\title{
Photometric Study of Radio Galaxies in the RATAN-600 "Cold" Survey
}

\author{
O. V. Verkhodanov, Yu. N. Parijskij, N. S. Soboleva, A. I. Kopylov, \\ A. V. Temirova, O. P. Zhelenkova \\ Special Astrophysical Observatory, Nizhnij Arkhyz, Russia
}

W. M. Goss

National Radio Astronomy Observatory, Socorro, NM, USA

\begin{abstract}
About 100 steep-spectrum radio sources from the RATAN$600 \mathrm{RC}$ catalog were mapped by the VLA and identified with optical objects down to $24^{m}-25^{m}$ in the $\mathrm{R}$ band using the 6-m telescope. An updated list of calibrators with known redshifts of the same class of RGs was compiled to evaluate the accuracy of photometric redshift estimates. BVRI photometry for $60 \mathrm{RC}$ objects was performed with the 6-m telescope, and by standard model fitting we have estimated color redshifts and ages of the stellar populations of the host $\mathrm{gE}$ galaxies. The mean redshift of FR II RGs from the RC list turned out to be $\approx 1$. Several objects were found in which active star formation began in the first billion years after the Big Bang.
\end{abstract}

A catalogue of 1145 radio sources has been obtained in the RATAN-600 "Cold" (RC) survey carried out in 1980-1988 with the radio telescope RATAN-600 in a strip of sky, $24^{h}$ long and $0^{\circ} .6$ wide $\left(\delta \approx+5^{\circ}\right)$, at 6 frequencies from 1 to 22 $\mathrm{GHz}$ with a sensitivity of about $3 \mathrm{mJy}$ at $3.9 \mathrm{GHz}$.

To select candidates for distant radio galaxies (RGs) in the "Big Trio" project (RATAN-VLA-SAO 6-m) we used the most distant population of radio sources in the 10-50 mJy range, where the $\log (N)-\log (S)$ curve has a maximum slope, and selected about 100 steep spectrum (SS) radio sources $(\alpha \geq 0.9)$.

In the following stages we did the selection of the FR II type RGs with VLA maps and optical identification and photometry from the 6-m telescope. 65 objects look like FR II and about 20 of them belong presumably to the most distant generation of RGs. 19 objects were classified as quasars by their stellar appearance on CCD images. Apart from the normal FR II RG class, some objects with SS happened to be of quite a different nature. 16 were not resolved even with the VLA in " $\mathrm{A}$ " configuration at $3.7 \mathrm{~cm}$, being less than $0^{\prime \prime} .2-1^{\prime} .0$ (CSS class). 16 "subgalactic" doubles, with sizes less than 4 ", are of separate interest. A few of them are very complex and cannot be considered as young progenitors of FR II objects.

The technique of multicolor photometry has became in the past few years the main method in selecting candidates for distant galaxies, and the only approach at very high redshifts. Determination of the age of high redshift stellar systems may be the only way of estimating the formation redshift of the first galaxies if star formation begins at redshifts larger than the $z$ of secondary 
ionization. Direct observation of the protogalaxies predicted by some recent computer simulations is not possible.

The method of multicolor photometry was checked on a sample of 45 radio galaxies published in the literature and carefully selected as steep spectrum high $z$ objects of the FR II type (Verkhodanov et al., 1999). It was shown that $B V R I$ colors are sufficient for accurate estimates of $z$ and age in the redshift range $0.5-3.5$.

We have implemented this approach for $60 \mathrm{FR}$ II and CSS radio galaxies of the RC catalogue that had been observed in $B, V, R_{c}, I_{c}$ bands in 1994-1998 with the CCD camera on the 6-m telescope. (Our sample of SS radio galaxies is now the largest one with four-band optical photometry.) The data were used to estimate color photometric redshifts and ages of host galaxies by comparison with the PEGASE (Fioc and Rocca-Volmerange, 1997) and GISSEL'98 (Bruzual and Charlot, 1993) models of evolution of the spectral energy distribution (SED). For a few typical cases, the reality of the color $z$ determinations was confirmed spectroscopically (Dodonov et al., 1999).

- It was shown that photometric redshifts give tolerable (10-20\% err) agreement with spectral ones for powerful RGs.

- A redshift-magnitude diagram shows much larger dispersion at $m_{R} \geq 22^{m}$.

- The distribution in $z$ of our objects has a maximum at $z \sim 1$, i.e., near the maximum activity stage in the Universe, and $\sim 10$ objects at $z>2.5$.

- A galaxy age is model-dependent and detected uncertainly (within the limits of $0.5-10 \mathrm{Gyr}$ ). One can set a lower limit to the galaxy age and, hence, $z$ of its formation. This age is always larger than the standard estimate of radio source lifetime.

The mean multicolor $(B V R I)$ age of the stellar population of the $\mathrm{RC}$ radio source host galaxies is of order $1 \mathrm{Gyr}$, and at least in some objects, active star formation had begun in the first Gyr after the Big Bang. Such distant objects must be of high density contrast, and modern cosmology has to explain this very early appearance of dense and massive (Tera-solar masses) protogalaxies, with quickly formed massive black holes inside them to produce FR II structures. From our 100 square degree area sample we can estimate that more than 10000 very early objects, born before the QSO epoch, are available on the sky and accessible for present-day optical and radio facilities. They can help us to penetrate into the "Dark Age" of the Universe, between the recombination epoch and the epoch of appearance of QSOs.

Future activity connected with the "Big Trio" project will be concentrated on direct spectroscopy of the most probable candidates for the first galaxy generation selected from our RC list.

\section{References}

Bruzual G. \& Charlot S. 1993, ApJ, 405, 538

Dodonov S.N., Parijskij Yu.N., Goss W.M., Kopylov A.I., Soboleva N.S., Temirova A.V., Verkhodanov O.V. \& Zhelenkova O.P., 1999, AZh, 76, 323.

Fioc M. \& Rocca-Volmerange B, 1997, A\&A, 326, 950

Verkhodanov O.V., Kopylov A.I., Parijskij Yu.N., Soboleva N.S. \& Temirova A.V. 1999. 